\title{
Miltefosine Susceptibility and Resistance in Leishmania: From the Laboratory to the Field
}

\section{Adriano C. Coelho*}

Departamento de Parasitologia, Instituto de Ciências Biomédicas, Universidade de São Paulo, São Paulo, 05508-900, SP, Brazil

\begin{abstract}
Miltefosine is the first effective oral drug used in the chemotherapy of leishmaniasis. The drug is more effective than pentavalent antimonials that are still considered as the drug of choice for treatment of leishmaniasis in several endemic regions. Efficacy rates of miltefosine against visceral leishmaniasis are up to $95 \%$, while against cutaneous leishmaniasis the rates vary between $53 \%$ and $91 \%$ depending on the species of the parasite and the endemic region. Recent reports have described an increased number of relapses in miltefosine-treated patients. This review describes the main findings associated with miltefosine susceptibility and resistance in Leishmania: two important factors involved in efficacy and failure in leishmaniasis treatment using this drug.
\end{abstract}

Keywords: Leishmania; Chemotherapy; Miltefosine; Drug resistance

\section{Introduction}

Species of genus Leishmania, the causative agents of leishmaniasis, are digenetic parasites transmitted by female sand flies that reside in cells of monocytic-phagocytic system of mammals as intracellular amastigotes. Parasites of genus Leishmania are classified into two subgenera: subgenus Leishmania (Leishmania) and subgenus Leishmania (Viannia). This subdivision is based on the localization of the promastigotes in the digestive tract of the sand fly. Around 20 species of the parasite are pathogenic to human and more than 30 species of sand flies are vectors of the disease. The disease has two patterns of transmission: anthroponotic and zoonotic. In the zoonotic leismaniasis, several mammalian species (wild or domestic) have been implicated as reservoir hosts of parasite.

The leishmaniases are responsible for two main clinical manifestations of the disease: tegumentary or visceral leishmaniasis (VL). In the visceral form, the parasite migrates to internal organs as the liver, spleen and bone narrow and may be lethal if not treated [1]. The tegumentary form may occur as ulcerative skin lesions developing at the site of the sand fly bite (localised cutaneous leishmaniasis [LCL]), multiple non-ulcerative nodules (diffuse cutaneous leishmaniasis [DCL]) or destructive mucosal inflammation (mucosal leishmaniasis [ML]) [2]. The clinical manifestation of each form of the disease depends on the species of infecting parasite and the host immunoinflamatory response. The disease occurs in tropical and subtropical regions of the world, particularly in Africa, Asia, America and in southern of Europe affecting about 12 million people worldwide with approximately 0.2 to 0.4 million VL cases and 0.7 to 1.2 million CL cases per year [3].

The chemotherapy for leishmaniasis is restricted in efficacy and in number of available drugs. Pentavalent antimonials are still the standard drug in some endemic areas despite its adverse reactions and progressive decreasing efficacy [4]. In the district of Bihar in India for example, dosages of the drug have been increased in the last decades, reaching the maximum acceptable toxicity [5]. Moreover, in this endemic region, reports show that up to $65 \%$ of patients do not respond to the treatment with pentavalent antimonials, indicating the emergence of drug resistant parasites [6-8]. An alternative to antimonials is amphotericin B. The liposomal formulation of amphotericin is widely used in Europe for example [9]. The cure rate can reach up to $95 \%$ of efficacy in patients with VL in just a single dose of the drug [10]. Despite its high toxicity, pentamidine is another alternative used in some endemic areas where antimonials are not more effective. Similar to the antimonials, pentamidine has significantly decreased its efficacy in more than $50 \%$ in the last decades. The drug is still used in some countries in South America against CL due to L. guyanensis $[11,12]$. The aminoglycoside paromomycin has recently approved for the treatment of VL in India [13]. Due to its low absorption in the gut, paromomycin requires parenteral administration, as it is also required for the other drugs mentioned before.

Miltefosine (MF) is the first and still the only oral drug used in the chemotherapy of leishmaniasis [14]. The drug is an analog of alkyl-lysophospholipid, initially developed against cancer. The antileishmanial activity was described in vitro and in vivo $[15,16]$ and some years later registered for the use in India against VL. The half-life of MF is between 150 and 200 hours leading to a sub-optimal concentration in the plasma, which can lead to the emergence of resistant parasites [17]. The main side effects of MF are transient gastrointestinal discomfort, vomiting, diarrhoea and increase of liver enzymes and serum creatinine [18]. MF is also teratogenic and therefore is strictly contraindicated in pregnant women.

Currently, the drug was already demonstrated as effective against VL and CL in South America [19,20]. MF has a rate cure of up to $95 \%$ against VL, with $100-150 \mathrm{mg} /$ day (or $2.5 \mathrm{mg} / \mathrm{kg}$ body weight) for 28 days $[14,21,22]$. On the other hand, cure rates for CL in South America vary between $53 \%$ and $91 \%$ in infections caused by different species of the parasite [23-26].

\section{Mechanism of action of Miltefosine}

The mechanism of action of MF is not completely understood and it is proposed that the drug has more than one target in the

*Corresponding author: Adriano C. Coelho, Departamento de Parasitologia Instituto de Ciências Biomédicas, Universidade de São Paulo, São Paulo, 05508 900, SP, Brazil, Tel: 55-11-3091-733; E-mail: ccadriano@gmail.com

Received January 20, 2016; Accepted February 04, 2016; Published February 11,2016

Citation: Coelho AC (2016) Miltefosine Susceptibility and Resistance in Leishmania: From the Laboratory to the Field. J Trop Dis 4: 203. doi:10.4172/2329891X.1000203

Copyright: (c) 2016 Coelho AC. This is an open-access article distributed unde the terms of the Creative Commons Attribution License, which permits unrestricted use, distribution, and reproduction in any medium, provided the original author and source are credited. 
parasite. The drug acts in cell membranes inhibiting phospholipid metabolism and the parasite's membrane composition by decreasing phosphatidylcholine and increasing phosphotidylethanolamine due to reduction of intracellular choline [27]. Additionally, changes in the length and level of unsaturation of fatty acids, as well as a reduction in ergosterol levels in promastigotes resistant to MF was found [28]. In the mitochondria, promastigotes treated with MF presented a significant reduction in the mitochondrial membrane potential and the enzyme cytochrome-c oxidase is inhibited in a dose dependent manner $[29,30]$. The drug binds to the outer leaflet of the plasma membrane and can be internalized by two possible mechanisms: (a) by endocytic pathway: the drug is internalized as member of an endocytic vesicle or (b) by flippase activity through the activity of an ATP-dependent mechanism mediated by the miltefosine transporter (MT) and its subunit Ros3 [31]. MT is an inward-directed lipid translocase that belongs to the P4 subfamily of P-type ATPases and Ros3 is a non-catalytic subunit of this transporter, which both play an important role in phosphocoline accumulation and in maintaining the phospholipid asymmetry of the parasite membrane [32-35]. Once accumulated, the drug can be eliminated by exocytosis or by floppase activity. Some members of $\mathrm{ABC}$ transporters subfamilies ( $\mathrm{ABCB}$ and $\mathrm{ABCG}$ ) can mediate this transport from the inner to the outer leaflet of the plasma membrane [36-38].

\section{Miltefosine susceptibility in Leishmania}

Molecular and biochemical differences among Leishmania spp. explain the differences in MF susceptibility. It is important to state that MF susceptibilities in promastigotes and intracellular amastigotes are correlated, what leads the choice of the promastigote form, that is easier to cultivate, for determination MF sensitivity in clinical isolates in most of the studies. An intrinsic difference in MF susceptibility is observed for different species and clinical isolates of parasite in vitro [35,39-42]. For example, among Leishmania spp. pathogenic to man, $L$. (L.) donovani is considered one of the most sensitive in both stages of the parasite, while $L$. (V.) braziliensis is the less sensitive $[40,42,43]$. A large variation in MF susceptibility is also observed in clinical isolates for several Leishmania species $[35,41,42]$. In $L$. (L.) amazonensis for example, in vitro tests against promastigotes and intracellular amastigotes showed that a clinical isolate was less susceptible to MF than a type strain of this species. The intrinsic tolerance to MF in this clinical isolate was not due to polymorphisms in the MT and Ros3 genes [35]. Moreover, this differential susceptibility in vitro did not either affect the clinical efficacy of infected mice with these two lines treated with MF [35]. Similarly, no correlation between the treatment outcome and MF susceptibility was observed in clinical isolates of $L$. (L.) donovani that presented large variation in drug sensibility [44]. Treatment with pentavalent antimonials has also demonstrated an ambiguous correlation between in vitro susceptibility of the parasite and treatment outcome in $L$. (L.) donovani and $L$. (V.) braziliensis infected patients $[45,46]$.

For drug activity against Leishmania spp., it is essential the internalization of MF and a clear correlation between drug uptake and susceptibility is observed [32,47,48]. As mentioned before, the accumulation of MF is mediated by the MT and its subunit Ros3 [31]. Interestingly, the activity and substrate specificity of this machinery vary between Leishmania species and may be correlated with the differential susceptibility of these species to the MF [48]. According to these authors, the reduced expression of the complex MT and its subunit Ros3 is responsible for the low sensitivity of $L$. (V.) braziliensis to MF [48].

The variation in MF susceptibility could also be explained by differences in rate of division of the parasites, exposure to the drug, biochemical targets and drug metabolism and biochemical content of the plasma membrane [31]. MF susceptibility is a trait that can be summarized therefore as drug tolerance that is an innate feature due to intrinsic biochemical and molecular properties of the parasite. Differently from drug tolerance, drug resistance is a feature that emerges and spreads after parasite being exposed to the drug.

\section{Miltefosine resistance in Leishmania}

Parasites resistant to MF can be obtained as promastigotes in vitro by increasing drug concentration (stepwise selection) $[32,35,41,49]$ or by chemical mutagenesis followed by selection of MF [32, 50]. In general, the mechanism of resistance is related to a defect in drug internalization due to mutations in the MT gene $[32,35,49,51,52]$. This defect in drug accumulation can be restored after functional expression of the MT gene in the resistant line [32,49]. Additionally, single mutations in Ros3 gene alleles were also observed in a MF resistant line leading to a high resistance level as observed in selected resistant lines containing mutations in MT alleles [33]. These data indicate that both proteins are selected during the drug pressure, although a higher recurrence of mutations in MT gene has been observed in resistant parasites [35,5153]. Besides, once the MT gene is inactivated, the resistance phenotype persists in amastigotes in vitro and in vivo in animal models of $\mathrm{VL}$ and CL $[35,54]$, indicating that this machinery is functional throughout the life cycle of the parasite. These findings showed that MT activity is essential for MF effectiveness and inactivation of this transporter becomes parasites completely refractory to MF.

Interestingly, when an alternative method using intracellular amastigotes in vitro to select MF resistant parasites, no change in MF susceptibility was found in amastigotes, although parasites were resistant when transformed back to promastigote $[55,56]$. The reason for this differential pattern of susceptibility in both stages of the parasite after MF selection in intracellular amastigotes is still unknown.

Miltefosine resistance can also be associated with an increase in efflux pumps through the overexpression of an $\mathrm{ABC}$ transporter. Some members of this family were already reported as able to mediated drug resistance: $A B C B 1$ from the subfamily $A B C B$ [38] and two members of the subfamily ABCG, ABCG4 and ABCG6 members $[36,37]$ are implicated in phospholipid trafficking and reduction in MF accumulation.

Recently, whole genome sequencing of two resistant lines of $L$. (L.) major revealed that inactivating mutations at conserved residues were able to confer MF resistance [53]. Mutations were also observed in the gene encoding previously pyridoxal kinase. Pyridoxal kinase plays a vital role in the formation of pyridoxal-5'-phosphate but the mechanism involved in MF resistance is still unknown [53]. Whole genome sequencing of $L$. major mutants resistant to $\mathrm{MF}$ also revealed a homozygous mutation in the $\alpha$-adaptin like protein gene in $L$. (L.) major and in two independent mutants of $L$. (L.) infantum [53]. This gene codes for the a subunit of Adaptor Protein 2 (AP2) complex involved in endocytosis of plasma membrane [57], suggesting a role in MF resistance in Leishmania. A role of this complex was already associated with suramin action and resistance in Trypanosoma brucei [58]. As mentioned before, MF may also enter in the parasite through the endocytic pathway, although this route is only important in a scenario in which the amount of drug bound to the membrane is extraordinarily high [31]. Overexpression of a functional copy of this gene in the resistant mutant and in wild-type parasites did not alter MF susceptibility [53]. Attempts to generate a double knockout of this gene were unsuccessful and no significant change in MF susceptibility was 
observed in single knockout parasites of L. (L.) donovani (unpublished observations). These findings indicate that these mutations may be related to fitness compensatory mutations, differently from mutations in the $M T$ gene that are directly involved in the primary resistance mechanism of the drug.

A global genomic expression by RNA microarray also revealed several differentially expressed genes in a MF resistant line of $L$. (L.) donovani, involved in DNA replication/repair mechanism, reduced protein synthesis and degradation, increased drug efflux, altered energy utilization and increased antioxidant defence mechanisms [51]. A point mutation was also found in the $M T$ gene of this resistant line [51]. Two clinically derived $L$. (L.) donovani strains with different inherent antimonial sensitivities were also selected in vitro as promastigotes and changes in the number of copies of chromosomes, single-base mutations and deletions of $M T$ gene were observed in these resistant lines [52]. Changes in the content of phosphatidylcholines and lysophosphatidylcholines were also associated with MF resistance in these two clinical lines [52].

As described above, most of knowledge about Leishmania MF resistance was performed in resistant parasites induced in vitro. Therefore, the molecular mechanisms and markers identified cannot be necessarily correlated with drug resistant parasites that emerge in endemic regions [59]. It might also be considered that anthroponotic disease in treated patients has a higher drug pressure on parasite population than the tegumentary disease in South America for example, that is zoonotic. All these aspects must be considered when clinical isolates from treated patients are studied. For example, a clinical decrease in the susceptibility of parasites to MF in vivo, a precursor of the emergence of drug resistance, has not yet been formally described, although several relapse cases after successful MF treatment have been reported for CL, DCL and VL [26, 60-65]. There is just one study that reports the selection of a $L$. (L.) infantum resistant line after treatment with MF in a HIV-coinfected patient [66]. The mechanism involved was associated with the occurrence of a mutation in the MT gene [66]. On the other hand, in patients infected with $L$. (L.) donovani, VL relapse cases were found up to $20 \%$ of patients after 6-12 months, but none of clinical isolates from these patients were resistant to MF in vitro [63]. Similarly, isolates of $L$. (L.) donovani from cured and failed patients showed a similar susceptibility to MF [43]. Otherwise, in clinical isolates of $L$. (V.) panamensis rescued before and after the treatment, an increase in MF susceptibility was found, indicating that resistant parasites were selected during the therapy with MF [41]. Finally, these studies indicated that the acquired resistance in leishmaniasis may or not be related with treatment failure using MF.

\section{Miltefosine treatment failure in the field}

As mentioned previously, the cure rate of MF in VL due to $L$. (L.) donovani is higher than $90 \%$, but in the last years, an increase in the number of relapses after the end of treatment has been reported [63,67]. In these studies, treatment failure was not due to intrinsic or acquired resistance and others factors recently reported in the literature have correlated relapse cases and treatment failure using MF, as for example: a lower exposure of the parasites to the drug (pharmacokinetics) [68], higher infectivity of the clinical isolates from these patients [69] and/or host-related factors (i.e. immunological factors) [70].

In South America, clinical studies for CL, show large efficacy variation between endemic regions and in infections caused by $L .(V$. braziliensis, L. (V.) guyanensis and L. (V.) panamensis [23-26]. Despite this variation in efficacy, recent clinical trials have shown that the therapy using MF is more efficacious than the standard therapy with meglumine antimoniate [23-25]. In DCL patients infected with either L. (L.) amazonensis or $L$. (L.) mexicana, a high clinical efficacy at the end of the treatment is observed, followed by relapses [60,65]. Similar findings with $L$. (L.) amazonensis were observed in mice infected and treated with MF [35]. Mice relapsed some months after treatment and no change in drug susceptibility was observed in parasites recovered from lesions, indicating that parasites did not acquire resistance to MF (unpublished observations). In tegumentary leishmaniasis, other factors unrelated to drug susceptibility and resistance may also be correlated to MF treatment failure, as for example: localization of the parasites in tissues less accessible to drugs, quiescence and presence of Leishmania RNA virus-1 (LRV1 virus) in the parasites of the Viannia subgenus [70,71]. Recent studies have correlated the prevalence of LRV1 virus in Leishmania (Viannia) species and treatment failure due to the subversion of the host immune response [71,72].

\section{Concluding Remarks}

Studies based on drug susceptibility and resistance have been useful to decipher the molecular basis involved in the mechanisms of action of drugs in Leishmania. MF is the only oral effective drug available for leishmaniasis. The drug has a long half-life (150-200 h) and the treatment is long ( 28 days) what can induce the emergence of resistant parasites in case of inadequate use. Understanding MF resistance is also a prerequisite to monitor drug resistant parasites in the field and preserve the drug efficacy. Currently, the main goal is to correlate the recent knowledge obtained in vitro and MF unresponsiveness in the field. MT and Ros3 might be considered as the main molecular markers in case of clinical isolates that show low MF sensitivity, since this machinery is essential for MF activity and efficacy. In the last years, an increase in the number of treatment failure cases has been reported for all the clinical forms of the disease and it is urgent to understand this increase in the number of cases. Recent reports have not directly correlated clinical inefficacy with intrinsic or acquired differences of drug susceptibility what indicates that other aspects are involved in MF inefficacy in the field.

\section{Acknowledgements}

The author acknowledges Dr. Cristiana T. Trinconi and Caroline R. Espada for critical reading of the manuscript.

\section{References}

1. Chappuis F, Sundar S, Hailu A, Ghalib H, Rijal S, et al. (2007) Viscera leishmaniasis: what are the needs for diagnosis, treatment and control? Nat Rev Microbiol 5: 873-882.

2. Murray HW, Berman JD, Davies CR, Saravia NG (2005) Advances in leishmaniasis. Lancet 366: 1561-1577.

3. Alvar J, Vélez ID, Bern C, Herrero M, Desjeux P, et al. (2012) Leishmaniasis worldwide and global estimates of its incidence. PLoS One 7: e35671.

4. Croft SL, Barrett MP, Urbina JA (2005) Chemotherapy of trypanosomiases and leishmaniasis. Trends Parasitol 21: 508-512.

5. Sundar S (2001) Drug resistance in Indian visceral leishmaniasis. Trop Med Int Health 6: 849-854

6. Lira R, Sundar S, Makharia A, Kenney R, Gam A, et al. (1999) Evidence that the high incidence of treatment failures in Indian kala-azar is due to the emergence of antimony-resistant strains of Leishmania donovani. J Infect Dis 180: 564 567

7. Sundar S, More DK, Singh MK, Singh VP, Sharma S, et al. (2000) Failure of pentavalent antimony in visceral leishmaniasis in India: report from the center of the Indian epidemic. Clin Infect Dis 31: 1104-1107.

8. Rijal S, Chappuis F, Singh R, Bovier PA, Acharya P, et al. (2003) Treatment of 
visceral leishmaniasis in south-eastern Nepal: decreasing efficacy of sodium stibogluconate and need for a policy to limit further decline. Trans R Soc Trop Med Hyg 97: 350-354.

9. Bern C, Adler-Moore J, Berenguer J, Boelaert M, den Boer M, et al. (2006) Liposomal amphotericin B for the treatment of visceral leishmaniasis. Clin Infect Dis 43: 917-924.

10. Sundar S, Chakravarty J, Agarwal D, Rai M, Murray HW (2010) Single-dose liposomal amphotericin B for visceral leishmaniasis in India. N Engl J Med 362: 504-512.

11. Lai A Fat EJ, Vrede MA, Soetosenojo RM, Lai A Fat RF (2002) Pentamidine, the drug of choice for the treatment of cutaneous leishmaniasis in Surinam. Int J Dermatol 41: 796-800.

12. Neves LO, Talhari AC, Gadelha EP, Silva Júnior RM, Guerra JA, et al. (2011) A randomized clinical trial comparing meglumine antimoniate, pentamidine and amphotericin B for the treatment of cutaneous leishmaniasis by Leishmania guyanensis. An Bras Dermatol 86: 1092-1101.

13. Sundar S, Jha TK, Thakur CP, Sinha PK, Bhattacharya SK (2007) Injectable paromomycin for Visceral leishmaniasis in India. N Engl J Med 356: 2571-2581.

14. Jha TK, Sundar S, Thakur CP, Bachmann P, Karbwang J, et al. (1999) Miltefosine, an oral agent, for the treatment of Indian visceral leishmaniasis. N Engl J Med 341: 1795-1800.

15. Croft SL, Neal RA, Pendergast W, Chan JH (1987) The activity of alky phosphorylcholines and related derivatives against Leishmania donovani. Biochem Pharmacol 36: 2633-2636.

16. Kuhlencord A, Maniera T, Eibl H, Unger C (1992) Hexadecylphosphocholine: oral treatment of visceral leishmaniasis in mice. Antimicrob Agents Chemother 36: $1630-1634$

17. Bryceson A (2001) A policy for leishmaniasis with respect to the prevention and control of drug resistance. Trop Med Int Health 6: 928-934.

18. Dorlo TP, Balasegaram M, Beijnen JH, de Vries PJ (2012) Miltefosine: a review of its pharmacology and therapeutic efficacy in the treatment of leishmaniasis. J Antimicrob Chemother 67: 2576-2597.

19. Jha SN, Singh NK, Jha TK (1991) Changing response to diamidine compounds in cases of kala-azar unresponsive to antimonial. J Assoc Physicians India 39 314-316.

20. Soto J, Toledo J, Gutierrez P, Nicholls RS, Padilla J, et al. (2001) Treatment of American cutaneous leishmaniasis with miltefosine, an oral agent. Clin Infect Dis 33: E57-61.

21. Sundar S, Jha TK, Thakur CP, Engel J, Sindermann H, et al. (2002) Oral miltefosine for Indian visceral leishmaniasis. N Engl J Med 347: 1739-1746.

22. Sundar S, Jha TK, Thakur CP, Bhattacharya SK, Rai M (2006) Oral miltefosine for the treatment of Indian visceral leishmaniasis. Trans R Soc Trop Med Hyg 100 Suppl 1: S26-33.

23. Soto J, Arana BA, Toledo J, Rizzo N, Vega JC, et al. (2004) Miltefosine for new world cutaneous leishmaniasis. Clin Infect Dis 38: 1266-1272.

24. Soto J, Berman J (2006) Treatment of New World cutaneous leishmaniasis with miltefosine. Trans R Soc Trop Med Hyg 100 Suppl 1: S34-40.

25. Machado PR, Ampuero J, Guimarães LH, Villasboas L, Rocha AT, et al. (2010) Miltefosine in the treatment of cutaneous leishmaniasis caused by Leishmania braziliensis in Brazil: a randomized and controlled trial. PLoS Negl Trop Dis 4: e912.

26. Vélez I, López L, Sánchez X, Mestra L, Rojas C, et al. (2010) Efficacy of miltefosine for the treatment of American cutaneous leishmaniasis. Am J Trop Med Hyg 83: 351-356.

27. Rakotomanga M, Blanc S, Gaudin K, Chaminade P, Loiseau PM (2007) Miltefosine affects lipid metabolism in Leishmania donovani promastigotes. Antimicrob Agents Chemother 51: 1425-1430.

28. Rakotomanga M, Saint-Pierre-Chazalet M, Loiseau PM (2005) Alteration of fatty acid and sterol metabolism in miltefosine-resistant Leishmania donovani promastigotes and consequences for drug-membrane interactions. Antimicrob Agents Chemother 49: 2677-2686.

29. Luque-Ortega JR, Rivas L (2007) Miltefosine (hexadecylphosphocholine) inhibits cytochrome $\mathrm{c}$ oxidase in Leishmania donovani promastigotes. Antimicrob Agents Chemother 51: 1327-1332.
30. Santa-Rita RM, Henriques-Pons A, Barbosa HS, de Castro SL (2004) Effect of the lysophospholipid analogues edelfosine, ilmofosine and miltefosine against Leishmania amazonensis. J Antimicrob Chemother 54: 704-710.

31. Pérez-Victoria FJ, Sánchez-Cañete MP, Seifert K, Croft SL, Sundar S, et al. (2006) Mechanisms of experimental resistance of Leishmania to miltefosine: Implications for clinical use. Drug Resist Updat 9: 26-39.

32. Pérez-Victoria FJ, Gamarro F, Ouellette M, Castanys S (2003) Functiona cloning of the miltefosine transporter. A novel P-type phospholipid translocase from Leishmania involved in drug resistance. J Biol Chem 278: 49965-49971.

33. Pérez-Victoria FJ, Sánchez-Cañete MP, Castanys S, Gamarro F (2006) Phospholipid translocation and miltefosine potency require both $\mathrm{L}$. donovan miltefosine transporter and the new protein LdRos3 in Leishmania parasites. $J$ Biol Chem 281: 23766-23775.

34. Weingärtner A, Drobot B, Herrmann A, Sánchez-Cañete MP, Gamarro F, et al. (2010) Disruption of the lipid-transporting LdMT-LdRos3 complex in Leishmania donovani affects membrane lipid asymmetry but not host cell invasion. PLoS One 5: e12443.

35. Coelho AC, Trinconi CT, Costa CH, Uliana SR (2014) In vitro and in vivo miltefosine susceptibility of a Leishmania amazonensis isolate from a patient with diffuse cutaneous leishmaniasis. PLoS Negl Trop Dis 8: e2999.

36. Castanys-Muñoz E, Alder-Baerens N, Pomorski T, Gamarro F, Castanys S (2007) A novel ATP-binding cassette transporter from Leishmania is involved in transport of phosphatidylcholine analogues and resistance to alkylphospholipids. Mol Microbiol 64: 1141-1153.

37. Castanys-Muñoz E, Pérez-Victoria JM, Gamarro F, Castanys S (2008) Characterization of an ABCG-like transporter from the protozoan parasite Leishmania with a role in drug resistance and transbilayer lipid movement Antimicrob Agents Chemother 52: 3573-3579.

38. Pérez-Victoria JM, Pérez-Victoria FJ, Parodi-Talice A, Jiménez IA, Ravelo AG, et al. (2001) Alkyl-lysophospholipid resistance in multidrug-resistant Leishmania tropica and chemosensitization by a novel P-glycoprotein-like transporter modulator. Antimicrob Agents Chemother 45: 2468-2474.

39. Fernández OL, Diaz-Toro Y, Ovalle C, Valderrama L, Muvdi S, et al. (2014) Miltefosine and antimonial drug susceptibility of Leishmania Viannia species and populations in regions of high transmission in Colombia. PLoS Negl Trop Dis 8: e2871.

40. Morais-Teixeira E, Damasceno QS, Galuppo MK, Romanha AJ, Rabello A (2011) The in vitro leishmanicidal activity of hexadecylphosphocholine (miltefosine) against four medically relevant Leishmania species of Brazil. Mem Inst Oswaldo Cruz 106: 475-478.

41. Obonaga R, Fernández OL, Valderrama L, Rubiano LC, Castro Mdel M, et al. (2014) Treatment failure and miltefosine susceptibility in dermal leishmaniasis caused by Leishmania subgenus Viannia species. Antimicrob Agents Chemother 58: 144-152.

42. Yardley V, Croft SL, De Doncker S, Dujardin JC, Koirala S, et al. (2005) The sensitivity of clinical isolates of Leishmania from Peru and Nepal to miltefosine. Am J Trop Med Hyg 73: 272-275.

43. Prajapati VK, Sharma S, Rai M, Ostyn B, Salotra P, et al. (2013) In vitro susceptibility of Leishmania donovani to miltefosine in Indian viscera leishmaniasis. Am J Trop Med Hyg 89: 750-754.

44. Hendrickx S, Eberhardt E, Mondelaers A, Rijal S, Bhattarai NR, et al. (2015) Lack of correlation between the promastigote back-transformation assay and miltefosine treatment outcome. J Antimicrob Chemother 70: 3023-3026.

45. Rijal S, Yardley V, Chappuis F, Decuypere S, Khanal B, et al. (2007) Antimonia treatment of visceral leishmaniasis: are current in vitro susceptibility assays adequate for prognosis of in vivo therapy outcome? Microbes Infect 9: 529-535.

46. Yardley V, Ortuno N, Llanos-Cuentas A, Chappuis F, Doncker SD, et al. (2006) American tegumentary leishmaniasis: Is antimonial treatment outcome related to parasite drug susceptibility? J Infect Dis 194: 1168-1175.

47. Pérez-Victoria FJ, Castanys S, Gamarro F (2003) Leishmania donovan Resistance to Miltefosine Involves a Defective Inward Translocation of the Drug. Antimicrob Agents Chemother 47: 2397-2403.

48. Sánchez-Cañete MP, Carvalho L, Pérez-Victoria FJ, Gamarro F, Castanys S (2009) Low plasma membrane expression of the miltefosine transport complex renders Leishmania braziliensis refractory to the drug. Antimicrob Agents Chemother 53: 1305-1313 
49. Coelho AC, Leprohon P, Ouellette M (2012) Generation of Leishmania hybrids by whole genomic DNA transformation. PLoS Negl Trop Dis 6: e1817.

50. Coelho AC, Trinconi CT, Senra L, Yokoyama-Yasunaka JK, Uliana SR (2015) Leishmania is not prone to develop resistance to tamoxifen. Int J Parasitol Drugs Drug Resist 5: 77-83.

51. Kulshrestha A, Sharma V, Singh R, Salotra P (2014) Comparative transcript expression analysis of miltefosine-sensitive and miltefosine-resistant Leishmania donovani. Parasitol Res 113: 1171-1184.

52. Shaw CD, Lonchamp J, Downing T, Imamura H, et al. (2015) In vitro selection of miltefosine resistance in promastigotes of Leishmania donovani from Nepal: genomic and metabolomic characterisation. Mol Microbiol.

53. Coelho AC, Boisvert S, Mukherjee A, Leprohon P, Corbeil J, et al. (2012) Multiple mutations in heterogeneous miltefosine-resistant Leishmania majo population as determined by whole genome sequencing. PLoS Negl Trop Dis 6: e1512.

54. Seifert K, Pérez-Victoria FJ, Stettler M, Sánchez-Cañete MP, Castanys S, et al. (2007) Inactivation of the miltefosine transporter, LdMT, causes miltefosine resistance that is conferred to the amastigote stage of Leishmania donovan and persists in vivo. Int J Antimicrob Agents 30: 229-235.

55. Hendrickx S, Boulet G, Mondelaers A, Dujardin JC, Rijal S, et al. (2014) Experimental selection of paromomycin and miltefosine resistance in intracellular amastigotes of Leishmania donovani and L. infantum. Parasitol Res 113: 1875-1881.

56. Hendrickx S, Inocêncio da Luz RA, Bhandari V, Kuypers K, Shaw CD, et al (2012) Experimental induction of paromomycin resistance in antimony-resistant strains of $\mathrm{L}$. donovani: outcome dependent on in vitro selection protocol. PLoS Negl Trop Dis 6: e1664.

57. McMahon HT, Mills IG (2004) COP and clathrin-coated vesicle budding: different pathways, common approaches. Curr Opin Cell Biol 16: 379-391.

58. Alsford S, Eckert S, Baker N, Glover L, Sanchez-Flores A, et al. (2012) Highthroughput decoding of antitrypanosomal drug efficacy and resistance. Nature 482: 232-236.

59. Maltezou HC (2010) Drug resistance in visceral leishmaniasis. J Biomed Biotechnol 2010: 617521.

60. Calvopina M, Gomez EA, Sindermann H, Cooper PJ, Hashiguchi Y (2006) Relapse of new world diffuse cutaneous leishmaniasis caused by Leishmania (Leishmania) mexicana after miltefosine treatment. Am J Trop Med Hyg 75: 1074-1077.
61. Ghosh S, Das NK, Mukherjee S, Mukhopadhyay D, Barbhuiya JN, et al. (2015) Inadequacy of 12-Week Miltefosine Treatment for Indian Post-Kala-Azar Dermal Leishmaniasis. Am J Trop Med Hyg 93: 767-769.

62. Pandey BD, Pandey K, Kaneko O, Yanagi T, Hirayama K (2009) Relapse of visceral leishmaniasis after miltefosine treatment in a Nepalese patient. Am J Trop Med Hyg 80: 580-582.

63. Rijal S, Ostyn B, Uranw S, Rai K, Bhattarai NR, et al. (2013) Increasing failure of miltefosine in the treatment of Kala-azar in Nepal and the potential role of parasite drug resistance, reinfection, or noncompliance. Clin Infect Dis 56: 1530-1538.

64. Soto J, Toledo J, Valda L, Balderrama M, Rea I, et al. (2007) Treatment of Bolivian mucosal leishmaniasis with miltefosine. Clin Infect Dis 44: 350-356.

65. Zerpa O, Ulrich M, Blanco B, Polegre M, Avila A, et al. (2007) Diffuse cutaneous leishmaniasis responds to miltefosine but then relapses. Br J Dermatol 156 1328-1335.

66. Cojean S, Houzé S, Haouchine D, Huteau F, Lariven S, et al. (2012) Leishmania resistance to miltefosine associated with genetic marker. Emerg Infect Dis 18 704-706.

67. Sundar S, Singh A, Rai M, Prajapati VK, Singh AK, et al. (2012) Efficacy of miltefosine in the treatment of visceral leishmaniasis in India after a decade of use. Clin Infect Dis 55: 543-550.

68. Dorlo TP, Rijal S, Ostyn B, de Vries PJ, Singh R, et al. (2014) Failure of miltefosine in visceral leishmaniasis is associated with low drug exposure. $J$ Infect Dis 210: 146-153.

69. Rai K, Cuypers B, Bhattarai NR, Uranw S, Berg M, et al. (2013) Relapse after treatment with miltefosine for visceral leishmaniasis is associated with increased infectivity of the infecting Leishmania donovani strain. MBio 4 e00611-00613.

70. Vanaerschot M, Dumetz F, Roy S, Ponte-Sucre A, Arevalo J, et al. (2014) Treatment failure in leishmaniasis: drug-resistance or another (epi-) phenotype? Expert Rev Anti Infect Ther 12: 937-946.

71. Adaui V, Lye LF, Akopyants NS, Zimic M, Llanos-Cuentas A, et al. (2016 Association of the Endobiont Double-Stranded RNA Virus LRV1 With Treatment Failure for Human Leishmaniasis Caused by Leishmania braziliensis in Peru and Bolivia. J Infect Dis 213: 112-121.

72. Bourreau E, Ginouves M, Prévot G, Hartley MA, Gangneux JP, et al. (2016) Presence of Leishmania RNA Virus 1 in Leishmania guyanensis Increases the Risk of First-Line Treatment Failure and Symptomatic Relapse. J Infect Dis 213: $105-111$. 